\title{
La práctica de actividades físicas y deportivas en el entorno del municipio y su implicación en el currículo de la educación física escolar The practice of physical activity and sport in the municipality of environment and their involvement in the curriculum school of physical education
}

\author{
Antonio BaenaExtremera*, GabrielFlores Allende**, Gabriel Barbero Montesinos*** \\ *IES Cardenal Cisneros (España), **Universidad de Guadalajara (México), ***Universidad Europea de Madrid (España)
}

\begin{abstract}
Resumen: El municipio se ha convertido en un importante proveedor de servicios a la comunidad, destacando la creciente oferta de actividades fisicodeportivas y recreativas, fomentando el deporte para todos los sectores de la población. En el presente artículo, pretendemos hacer una reflexión sobre los recursos que nos ofrece el municipio, la gestión de estas prácticas deportivas y las implicaciones curriculares que nos permiten en la enseñanza obligatoria.
\end{abstract}

Palabras clave: Actividad Física, Actividad Deportiva, Municipio, Currículum.

Abstract: The municipality has become an important provider of services to the community, having emphasized the increasing range of physical activities and recreational sports, promoting the sport to all sectors of the population. In this article, we intend to reflect on the resources we offer the municipality, the management of sports and the curricular implications that allow us in obligatory education.

Key words: Physical activity, Sport activity, Town, Curriculum.

\section{Introducción.}

Se entiende por Municipio, a la persona de Derecho Público, constituida por una comunidad humana, asentada en un territorio determinado, que administra sus propios y determinados intereses, y que depende siempre, en mayor omenor grado, de una entidad pública superior: el Estado nacional o provincia (Jasso, 2000, citado en Ruiz Munuera, 2002).

El municipio se ha convertido en un importante proveedor de servicios a la comunidad. Entre otros servicios, destaca la creciente oferta de actividades físicas, deportivas y recreativas, el patrocinio de pruebas o de deportistas individuales y/ó deportivos de toda índole, de fomento del deporte para todos (campañas de natación, de gimnasia, de mantenimiento para adultos, de aeróbic, día de la bicicleta, carreras populares, street basket, etc.) en especial en los municipios con cierta población.

Un indicador de que la evolución en los últimos años de los servicios municipales de deportes ha sido positiva, son los siguientes aspectos (Rodríguez, 2003):

1.La preparación, experiencia y el nivel de profesionalización de los técnicos que vienen desarrollando labores de gestión y organización de actividades deportivas en el ámbito municipal.

2.La formación específica y el intercambio de experiencia que se han venido desarrollando a través de cursos y seminarios de trabajo de gestión deportiva, que han complementado la escasa formación inicial. 3.La utilización de nuevas tecnología que nos han ayudado a mejorary sacar más provecho a nuestra actuación profesional.

4.La implicación de los poderes públicos, especialmente la administración municipal por su cercanía, en la promoción directa e indirectamente (mediante subvenciones o prestamos de instalaciones) de las diferentes posibilidades de actividad física (lúdica, educativa, iniciación deportiva, mantenimiento, competición, etc.) entre los diversos estratos de la ciudadanía (niños, jóvenes, adultos, minusválidos, mayores, etc.).

Si realizamos una revisión profunda sobre el papel que estos profesionales vienen desarrollando en sus labores relacionadas con la gestión deportiva en el ámbito municipal, podemos deducir una serie de principios generales de actuación:

La oferta de instalaciones y actividades se desarrollará básicamente para satisfacer la demanda existente, si bien deberemos prever un posi-

Fecha de recepción: 07-09-06 - Fecha de aceptación: 25-11-06

Correspondencia: Antonio Baena Extremera

C/ Granada, 6

18217 Güevéjar (Granada)

E-mail: abaenaextrem@yahoo.es ble aumento de la misma, derivada de la planificación y programación de actividades a desarrollar en un futuro por parte de los servicios municipales de deportes. Por ejemplo, no es infrecuente la construcción de equipamientos deportivos sin una necesidad previa ni una planificación futura que justifique dichos equipamientos, sin tener un conocimiento de la realidad social, empleando como justificación unas elecciones o el capricho político.

El deporte y la actividad física deben forma parte de un sistema integral socio-económico, en el que todos sus componentes se relacionen de forma interactiva. Por ejemplo: unos equipamientos deportivos deben estar pensados hacia y desde la socialización, no sólo hacia el uso exclusivamente deportivo ó competitivo. Deben tener un carácter multiuso y que atienda a las diferentes necesidades de la población.

Debemos prever el aumento de práctica motivado por la extensión de la Educación Física escolar, considerado que ésta tenga como uno de sus fines el desarrollo de unos hábitos de práctica de la actividad física. Por ejemplo: será un objetivo básico de la EF en la etapa escolar, desarrollar hábitos hacia la actividad física entre los escolares, potenciando y valorando la participación en horario extraescolar en actividades físicodeportiva en instalaciones municipales y/o promovidas por el municipio, como un servicio fundamental a la comunidad.

No obstante, nos encontramos ante unos problemas de ajuste económico que de alguna forma pueden modificar el actual modelo de Servicio Público benefactor, a otro más restrictivo a la hora de distribuir recursos, debido al incremento de nuevos equipamientos deportivos en los últimos años, que a su vez requiere de un incremento presupuestario dirigido hacia el mantenimiento y dotación de personal de esas instalaciones.

Una posible solución de dicha problemática, sobre todo en cuanto a la optimización de los recursos, puede ser el establecimiento de una nueva política de descentralización de recursos a través de un modelo de Gestión Indirecta, en el que puedan llegar a participar los propios centros escolares, lo cual implica potenciar el tejido asociativo (clubs deportivos, asociaciones de alumnos, APA, etc.) y dotarle de medios a nivel de:

-Recursos económicos y/o materiales.

-Personal técnico especialmente formado en tareas de promoción, gestión y organización deportiva.

-Instalaciones, manteniendo una relación de ayuda y colaboración hasta conseguir una capacidad de gestión propia.

\section{Competencias legislativas a nivel municipal.}

A la hora de hablar de prácticas físico-deportivas en el municipio, localidad o ciudad es importante tener en cuenta la siguiente legislación. 
Carta Europea del Deporte para todos: Los ministros europeos reunidos en Bruselas en Marzo de 1975 y revisado en Marzo de 1979, destacan el derecho de todo individuo a la práctica del deporte y la obligación de los gobiernos a fomentar la creación de estructuras nacionales para el desarrollo y la coordinación del Deporte para todos.

Por otro lado, en 1978, se proclamó la Carta Internacional de la Educación Física (EF) y el Deporte de la UNESCO, durante la cual se creó el Comité Intergubernamental para la Educación Física y el Deporte. En esta carta se aboga por el derecho fundamental de todo ser humano de acceder a la Educación Física y al Deporte. Además, reconociendo que son fundamentales para el pleno desarrollo de su personalidad, y siendo necesarias unas instalaciones adecuadas y reconociendo a los poderes públicos como favorecedores de las actividades fisicas y deportivas cuyo valor educativo sea manifiesto.

A nivel estatal, destacar:

Art. 43.3 de la Constitución Española: Los poderes públicos fomentarán la educación sanitaria, fisica y deportiva, considerando a ésta última como una actividad popular y para todos...»

Ley de Bases del Régimen Local: El municipio ejercerá competencia en materia de actividades e instalaciones deportivas, ocupación del tiempo libre (Art. 24.2).

Ley 13/1980 de 21 de Marzo (modificada en 5 de Octubre de 1990) de Cultura Física (Art. 3):

a) Desarrollar la política fisico-deportiva o gestionar la utilización de las instalaciones públicas.

b) Llevar un censo de las instalaciones deportivas, así como de su estado de conservación dotándolas de personal adecuado.

c) Asegurar el cumplimiento de la legislación urbanistica en materia de reserva de espacios y zonas para la práctica del deporte.

d) Celebrar concierto y convenios con entes públicos y privados para el cumplimiento de los fines de la presente ley.

Ley Reguladora de Bases del Régimen Local de 2 de Abril de 1985, donde se enmarcan y definen las obligaciones de los Municipios. En su Art.25.1: El Municipio, para la gestión de sus intereses y en el ámbito de sus competencias, puede promover toda clase de actividades y prestar cuantos servicios públicos contribuyan a satisfacer las necesidades y aspiraciones de la comunidad vecinal. En el punto 2 sigue diciendo: El Municipio ejercerá competencias en Actividades o instala ciones culturales y deportiva; ocupación del tiempo libre; turismo, etc. También en el Art. 26 se dice: Los Municipios por sí o asociados, deberán prestar los servicios siguientes: en los municipios con pobla ción superior a 20.000 ha, ....instalaciones deportivas de uso público.

Ley del Deporte (Ley 10/1990): El Art. 2 de la Ley 10/1990 de 15 de Octubre establece que la administración del estado ejercerá las competencias atribuida por esta ley y coordinará con las C.C.A.A. yen su caso con la C. Locales aquellas que puedan afectar directa y manifiestamente a los intereses generales del deporte en el ámbito regional,... y se traslada a las provincias y a las Administraciones Locales que tengan de 20.000 hab. contando con los medios y servicios que las C.C.A.A. les traspase.

El capítulo III de la Ley 9/1990, se refiere a las competencia de las Entidades Locales. El Art.23 afirma que las entidades locales desarrollarán las siguientes competencias:

1.La elaboración y ejecución de los planes necesarios para dotar al municipio de la superficie para una infraestructura deportiva.

2.La gestión de los equipamientos deportivos municipales, sin perjuicios de los conciertos y convenios que puedan celebrar con entidades públicas o privadas.

3.Asegurar el cumplimiento de la legislación urbanística en materia de reserva de espacios y zonas para la práctica deportiva.

4.Ejercer cuantas funciones y competencia les estén atribuidas en virtud de la normativa estatal de la presente ley de las que se desarrollen, asi como las que sean transferidas o delegadas.

5.Los municipios en el ejercicio de sus competencias y dentro de sus respectivos ámbitos territoriales, podrán elaborar y ejecutar sus programas de promoción deportiva, sin perjuicio de las facultades de coordinación que correspondan a la administración.
Se transfieren a los municipios de 20.000 hab. la titularidad y la gestión de las instalaciones deportivas que son propiedad de las C.C.A.A. y se encuentren en el correspondiente término municipal. Asimismo, algunas C.C.A.A. pueden permitir a dichos municipios:

- Organizar las competiciones escolares dentro de sus respectivos ámbitos territoriales.

- Promover la creación de agrupaciones deportiva para el desarrollo del deporte escolar, especialmente en las zonas social y económicamente desfavorecidas.

- Gestionar la concesión de ayudas para crear y mantener equipos y para actividades deportivas en los centros escolares.

- La promoción del deporte social y popular.

- La autorización y en su caso organización de manifestaciones deportivas populares.

- La promoción y creación de agrupaciones deportivas para el desarrollo del deporte para todos y del deporte de tiempo libre.

3. Modelos y propuestas de actuación físico-deportiva en el entorno del municipio.

Podemos diferenciar distintos modelos de actuación deportiva municipal en cuanto a la promoción de actividades físico-deportiva, en función de las prioridades establecidas y de la asignación presupuestaria que reciben los diferentes programas:

a) Ayuntamiento que centra su actuación deportiva en el fomento de la EF. En estos casos, este es el programa con más presupuesto.

b) Ayuntamientos que centra su actuación deportiva en programas de natación. En bastantes poblaciones donde el municipio gestiona una piscina cubierta, las actividades acuáticas son prioritarias.

c) Ayuntamientos que centran su actuación en el deporte escolar y/o en las escuelas deportivas.

d) Ayuntamientos que centran su atención en los programas de ocio y recreación.

e) Ayuntamientos que fomentan programas de deporte-salud o asistenciales.

f) Ayuntamientos que promocionan la práctica deportiva de actividades alternativas o de contacto y disfrute del medio natural.

Los posibles agentes promotores de la EF y el Deporte en los municipios pueden ser (Rodríguez, 2003):

a)Instituciones:

1.Escolares: Educación Infantil, Primaria, Secundaria, Universidad,Adultos.

2.Organismos Públicos: Ayuntamientos, CCAA, Diputaciones, Otros.

3.Entidades Privadas:

*Entidades Deportivas: Clubs, Federaciones, Escuelas de Inicia-

ción,...

*Empresas Deportivas: Gimnasios, Expresas de Servicios,...

*Empresas no Deportivas: Cajas, Comercios, ...

b)No Instituciones: Familia, Individuo.

Algunos municipios recogen varios modelos de actuación según sus prioridades o utilizan combinación de los mismos. La tendencia de muchos municipios, sobre todo de las grandes ciudades, se está centrando en un proceso de traspaso a los agentes sociales (clubs, asociaciones, ...) en la gestión de los programas que eran ejecutados inicialmente desde el municipio directamente o a través de una fundación, patronato municipal, etc. El tejido asociativo enriquece y favorece la difusión de las actividades de una manera equilibrada y respondiendo siempre a los intereses de los ciudadanos. En esta línea, la estrategia municipal se basa en ayudas para la vertebración de estas entidades que dan vida y dinamismo a las ciudades. Por otra parte, evita un dimensionado en instalaciones, recursos y medios humanos que pueden rebasar la capacidad económica y administrativa del ente municipal que gestiona el deporte en dicho ámbito, e incluso del propio municipio.

En la actualidad instituciones públicas o promotores privados realizan significativas inversiones en el sector de la AFD con diferentes fines de tipo social-empresarial, lo que unido a un marco legislativo protector-promotor y a la existencia de creciente demanda, produce 
ofertas que justifican la especialización de aquellos profesionales y sistemas que atienden al sector. Organizaciones diferentes enfatizarán más unos objetivos que otros, bien por su propia naturaleza constitutiva, bien por la definición de sus objetivos (De Lucas, 2005).

Un servicio municipal orientará su atención preferente a jóvenes, mayores, discapacitados; a la democratización de prácticas orientadas a la salud, la formación y los valores básicos de convivencia social; Federaciones, clubes, ..., a la obtención del mayor rendimiento deportivo por parte de sus mejores especialistas para captar la atención del mayor número de espectadores; las empresas a la obtención de beneficios. En todos los casos con necesidad de dirección especializada que oriente y garantice la consecución de objetivos, de manera eficiente económicamente y fiable desde el punto de vista de la calidad. Para conseguir esto último, siguiendo la adaptación del modelo de evaluación de la European Foundation for Quality Management, realizada por el Ministerio de Administraciones Públicas y haciéndola extensiva a cualquier tipo de organización, han de considerarse características como las siguientes (De Lucas, 2005):

- Importancia de las personas.

- Orientación al cliente (ciudadano).

- Reconocimiento (a personas en su trabajo).

- Gusto por el trabajo bien hecho.

- Trabajar con hechos y datos.

- Relaciones de asociación (entre unidades, con clientes, proveedores...) - Ética.

- Orientación a resultados (búsqueda de eficacia, consecuencia de la atención a las necesidades).

Vamos a dividir las actividades en dos grandes apartados, siguiendo la propuesta de Carranza (1990, citado en Rodríguez, 2003):

1.Actividades de ámbito escolar:

*Escuela:

- Jornadas de toda la escuela: Día de la actividad física, fiesta del agua, fiesta del juego popular, juegos de cooperación con todo el mundo.

- Iniciación y promoción deportiva con soporte exterior: Tenis de mesa, esgrima, voleibol, bádminton, ...

- Competiciones escolares: Entre alumnos del mismo centro, alumnos de otros centros,...

*Ciudad:

- Actividades puntuales entre escuelas: Bailes, encuentros atléticos,...

- Visitas e itinerarios: Itinerarios por zonas de la ciudad, visita a instala-

ciones, ...

- Iniciación deportiva en instalaciones específica: Modalidad de patinaje, atletismo, actividades acuáticas, vela, escalada, hípica,...

- Uso de espacios de libre acceso desde el centro docente: Sesión de EF fuera del centro, recorridos bicicleta de montaña, orientación, escalada... - Participación en actos puntuales de la ciudad: Carrera de patines, manifestaciones en bicicleta de montaña, carreras populares, etc.

*Naturaleza:

- Colonias deportivas, colonias.

- Itinerarios y marchas.

- Jornadas en la naturaleza.

2. Actividades de ámbito no escolar

2.1. No Competitivas.

2.1.1. Oferta de uso no programado:

* Actos puntuales:

- Carrera de recorrido: Carreras populares, carrera de patrocinador, parque de la bicicleta, carrera popular de patines, etc.

- Actividades recreativas: Salón de la infancia y de la juventud, fiesta del deporte, parques de atracciones, ferias populares, etc.

- Espectáculos: Espectáculos deportivos, circo, teatro, etc.

*Actividades continuadas:

- Museos, visitas, exposiciones: Museo del deporte, museo de entidad o club deportivo, museo arqueológico, parque de las ciencias,...

- Espacios donde se promueve la libre práctica: parque y zonas de juegos, zonas de monopatín, carriles de bicicletas, ludo tecas, etc.

*Actividades de temporada:
-Actividades acuáticas: Piscinas municipales, la playa en verano, zonas de baño, pantanos,...

- Campañas de nieves: Cursos del patronato y federativos, etc.

2.1.2. Oferta de uso programado:

-Actividades polivalentes:Actividades extraescolares para alumnos del centro.

- Actividades extraescolares abiertas a personas ajenas al centro; excursionismo.

-Actividades univalentes: Iniciación deportiva, cursos de natación, perfeccionamiento de un deporte, etc.

- Actividades de temporada: Campañas de verano, estancias de tecnificación, salidas a la nieve.

2.2. Competitivas.

- Competiciones deportivas extraescolares de carácter escolar: Individuales, de adversario, etc.

- Competiciones federativas.

- Estancias de tecnificación.

- Centros de alto rendimiento deportivo.

\section{Recursos Materiales y Humanos en las prácticas físico- deportivas en el municipio.}

Elárea de EF puede utilizar tales recursos comunitarios, unas veces dentro del propio centro escolar y otras trasladándose hacia instalaciones o parajes próximos que proporcionan a los alumnos y alumnas experiencias ajenas a la rutina diaria (Baena, 2005).

Debemos partir del dato evidente que la situación de los equipamientos e instalaciones deportivas municipales están en continuo crecimiento, no obstante para conocer el número y sus características nos podemos remitir al estudio realizado por el C.S.D. (1987) donde se indicaba que:

- A nivel nacional el número de instalaciones públicas y privadas es de 484.9997, es decir, 19,7 por cada 10.000 hab.

- El $50 \%$ de las instalaciones son municipales.

- El $40 \%$ que practica deporte utiliza instalaciones municipales, siendo el hombre más que la mujer.

- El uso de instalaciones desciende con la edad. El uso aumenta con el nivel de estudios.

En otro estudio realizado más recientemente, en censos de instalaciones nacionales promovidos por el Consejo Superior de Deportes se totalizan 48.205 instalaciones con 99.775 espacios deportivos en 1986; 66.353 instalaciones con 153.635 espacios en 1997 se calcula que el censo previsto para 2005 compute más de 80.000 instalaciones en toda España con unos 200.000 espacios (De Lucas, 2005).

Una vez visto la disponibilidad de los RRMM, a nivel general, y siguiendo las indicaciones de Baena (2005), los recursos de la Comunidad o Municipio sería:

A) Recursos materiales de préstamo o alquiler: Nos referimos a aquellos materiales que el profesorado y los centros pueden utilizar $\sin$ necesidad de adquirirlos. Provienen de entidades, servicios y organismos públicos y/o privados que ofrecen apoyo a los centros educativos o con los que se pueden establecer convenios o acuerdos de cooperación: Centros de profesores, Clubes deportivos, Centros de iniciación y perfeccionamiento deportivo, Consejería de Educación y Ciencia, Consejería de Turismo y Deporte...

B) Recursos ambientales: Donde vamos a distinguir dos tipos:

B.1. Paisaje urbano: Rebollo (1996) efectúa un análisis de los diferentes recursos que cualquier localidad puede aportar para la práctica de actividad fisico deportiva. Tales medios, pertenecientes a la comunidad (Ayuntamientos, Diputaciones, Asociaciones...) y que pueden ser aprovechados por nuestros escolares durante las actividades lectivas, complementarias o extraescolares, son de naturaleza diferente en función del tamaño y del número de habitantes. Siguiendo este criterio establece una clasificación o agrupación en cuatro niveles o escalones para descubrir, mediante ella, el tipo de recursos predominantes en cada municipio. 
1.Poblaciones pequeñas:

-Pistas polivalentes, generalmente en mal estado y en algunos casos inutilizadas por falta de personal de mantenimiento y técnico.

-Medio natural, cuya proximidad potencia la realización de actividades como senderismo, cicloturismo o escalada.

2. Poblaciones medianas: Son poblaciones, cabecera de comarca, donde el desarrollo económico es mayor y consecuentemente donde también aumentan las posibilidades de utilización de los recursos del entorno: Polideportivos al aire libre y cubiertos, Campo de fútbol, Piscina al aire libre, Zonas verdes de juego y recreo.

3. Ciudades: En las ciudades hay un aumento en la oferta de recursos y, consecuentemente, se incrementan las posibilidades que los centros escolares tienen para hacer uso de instalaciones y equipamientos ajenos al entorno escolar. A pesar de ello disminuyen mucho las oportunidades para contar con equipamientos en zonas naturales quedando reducido el espacio natural útil a zonas de parques y paseos.

4. Las grandes ciudades: Las grandes ciudades cada vez se preocupan más por ofrecer espacios y opciones urbanísticas que promuevan el desarrollo y práctica de actividad física y deportiva. Contamos con algunos ejemplos que pueden servir para demostrar este interés.

-Itinerarios fisico-turísticos para peatones. Es una idea surgida en la ciudad de Zurich. Consistía en proporcionar planos de los diferentes barrios de la ciudad en los que se ofrecían recorridos indicando: tiempo de recorrido, zonas recreativas, zonas deportivas...Como variante a esta actividad surge la orientación urbana (a pie, en bicicleta...).

- Los campos de juego. Son zonas naturales y espacios abiertos de infinitas posibilidades motrices. Se componen de una serie de áreas:

- Terrenos para niños pequeños (recintos de arena, estanques de chapoteo)

- Terreno con toboganes, balancines, juegos de construcción, vehículos reales, aparatos de trepa y serpenteo...

- Explanadas para los juegos y deportes.

- Anfiteatro natural.

- Zonas de juego y deportes tradicionales para adultos.

- Pistas para bicicleta y actividades atléticas...

- El terreno de aventuras. El CODEJ (comité francés para el desarrollo de los campos de juego) define el terreno de aventuras como: «Es el terreno indefinido, la 'zona' o el bosquecillo en el que durante la infancia los niños construyen su cabaña, hacen sus hoyos, transportan piedras y ramas, trepan por los árboles... entre los requisitos que deben cumplir los terrenos de aventuras destaca su proximidad (menos de 500 metros) a las residencias de los niños, escuelas o guarderías».

- Los parques Trimm. Son parques naturales o urbanos acondicionados para la práctica física. Se estructuran en las siguientes partes: Una pista Trimm para carrera, un campo Trimm para lograr efectos sensomotores de movilidad y fuerza, un campo de juegos con objetivos de socialización, unas cabañas Trimm (vestuarios, servicios, sauna, almacén...)

- Espacios acuáticos. Son un lugar idóneo para contactar con el agua, con un fin educativo.

B.2.Paisaje natural: El medio natural es un lugar cada vez más demandado para el desarrollo de actividades físicas y deportivas. Este paisaje situado, normalmente, muy alejado de los centros escolares, también puede ser un recurso de gran potencialidad educativa cuyo aprovechamiento debe proyectarse con el fin de acercar a los alumnos y alumnas a la naturaleza.

Existen, por tanto, diferentes tipos de equipamientos, de titularidad diversa (pública, asociaciones, empresas de ocio y tiempo libre...) que ofrecen grandes oportunidades educativas. Algunos son de carácter natural y otros creados por el hombre en la naturaleza para cumplir, entre otras, con esta finalidad. Ejemplos serían:

- Equipamientos de carácter natural: Zonas montañosas, senderos, veredas y caminos forestales, nieve, ríos, embalses, pantanos, lagos, mar...

- Equipamientos creados por el hombre en la naturaleza. Áreas de acampada, infraestructuras para la práctica del esquí, aulas de la naturaleza, circuitos vita, terrenos de aventuras, pistas finlandesas...
- Los circuitos vita: Circuito creado en los años sesenta en Suiza con la idea de realizar práctica física en zonas verdes. Consiste en un itinerario de 20 estaciones, con un recorrido de tres kilómetros donde se especifica por medio de postes y paneles informativos la actividad que se debe realizar en cada uno de ellos. Este proyecto fue financiado por una casa de seguros llamada «Vita» y por eso, en un principio, se denominó así. Posteriormente, tales circuitos, han ido adoptando diferentes nombres en función de las empresas que han financiado su construcción. Así, en España, el mismo circuito se conoce con el nombre de «Circuitos Cola-Cao». Los aparatos utilizados suelen ser: artificiales, procedentes del medio o bien utilizando el cuerpo como elemento de trabajo. Comienza con una carrera de 200 a 300 metros, seguida de ejercicios variados gimnásticos y finalmente termina, de nuevo, con carrera continua. Se determina en cada sesión el número de repeticiones según las edades.

En relación a los recursos humanos, para intentar clarificar la ingente cantidad de recursos que, pertenecientes a la comunidad, la escuela puede demandary utilizar, realizaremos la siguiente clasificación de los Recursos Humanos de los Municipios:

A) Voluntariado: La orden 11 de noviembre de 1997 (BOJA 2711-97) tiene por objeto promover e impulsar la participación solidaria de los ciudadanos en el ámbito educativo mediante actuaciones de Voluntariado y a través de entidades colaboradoras. Para ello las entidades que deseen llevar a cabo tales actividades de colaboración en un centro educativo, deberán presentar un «proyecto», a la dirección del mismo, para ser aprobado por el Consejo Escolar.

Desde el punto de vista del área de EF estas acciones de colaboración, centradas en el horario extraescolar, podrían servir para aumentar la oferta de actividades físico-deportivas al poder contar con personas comprometidas en las tareas educativas del segundo tiempo pedagógico.

B) Padres y madres: Las asociaciones de padres, reguladas en Andalucía a través del Decreto 27/1988, contienen entre sus metas la colaboración con los centros en la realización de actividades complementarias y extraescolares y organizar actividades culturales y deportivas. Es evidente como los padres y madres, pueden coparticipar en la celebración y organización de encuentros, actividades o torneos deportivos y así colaborar en la formación total de sus hijos e hijas.

C) Otros recursos humanos: Los centros educativos pueden contratar servicios, con los límites que la Consejería de Educación y Ciencia establezca. Esto, que aparece regulado en el Decreto 200/1997, de 3 de septiembre, por el que se aprueba el Reglamento Orgánico de las IES, puede suponer, por ejemplo, la presencia de monitores deportivos en el centro, siempre bajo la tutela y dirección del maestro o maestra especialista en EF.

También las entidades locales, Ayuntamientos y Diputaciones, pueden colaborar con los centros en la realización de actividades extraescolares. Así, son numerosas las experiencias deportivas que los niños y niñas acometen y que son dirigidas por personal especialista contratado o perteneciente a tales organismos.

Igualmente la propia Consejería de Educación y Ciencia y otras como la de Turismo y Deporte proponen, anualmente, la posibilidad de realizar múltiples actividades deportivas que subvencionadas son, también, dirigidas por personal especialista.

La situación laboral y técnico-profesional del personal técnico de los servicios municipales de toda España, no era la más adecuada para favorecer un desarrollo óptimo de las políticas deportivas municipales. Tanto por su nivel de formación y titulación como por su retribución económica, las características de dicho personal en general no nos permitían crear unas grandes expectativas de futuro para el desarrollo óptimo de dichos programas.

Nos remitimos al estudio de Martínez y cols. (1991) sobre la estructura ocupacional del deporte en España, en el que se detallan aspectos relacionados con los profesionales de la actividad física y el deporte, que desempeñan su labor profesional en las administraciones municipales a través de sus servicios de deportes. 


\section{Responsables en gestión deportiva.}

Titulación: El 50\% de los contratados no poseen una titulación específica, para desarrollar dicha labor. El $33 \%$ poseen un título federativo-deportivo, por lo tanto tampoco cuentan con una titulación adecuada para sus funciones. Por último, tan sólo un $10 \%$ poseen la titulación de Licenciado en Ciencias de la Actividad Física y del Deporte, que sin ser específica, si es la más próxima a las labores de organización y gestión.

\section{Dirección técnico-Deportiva.}

Titulación: En este caso el 50\% corresponde con titulaciones específicas de Licenciado en Ciencias de la Actividad Física y del Deporte. Por un 30\% de títulos federativos, con menor calificación técnico-profesional. Y por último sin ninguna cualificación, ni formación se encuentran el $23 \%$ de los contratados.

\section{Monitores Deportivos.}

Titulación: El 50\% de los técnicos que actualmente poseen los servicios deportivos municipales son monitores deportivos, con un perfil profesional no debidamente adecuado para la labor que este colectivo debe desarrollar dentro de este tipo de servicios. Dentro de las características de estos profesionales podemos destacar que son:

- Jóvenes solteros entre los 20-34 años en el 70\% de los casos.

- Sólo el $15 \%$ son mujeres.

- La situación de empleo es bastante precaria, pues la mayoría están bajo contratos temporales, no superiores a las $20 \mathrm{~h}$ semanales.

- En el 75\% de los casos sus ingresos son inferiores a los 300 euros.

- Para la mayoría su antigüedad en las entidades contratadas no superan $\operatorname{los} 3$ años.

-Su titulación es exclusivamente federativa.

D) Orientadores: Aquí el protagonismo recae sobre los docentes especialistas que quieren hacer uso de la formación permanente institucional y se acogen a los servicios que desde los centros de profesores el personal orientador diseña para ellos.

\section{Implicación en el currículo de la Educación Física escolar}

Se considera que los profesores de $\mathrm{EF}$ en $u n n^{\circ}$ significativo limitan su actividad docente al horario escolar, si bien esto no es así en la totalidad de los casos. Uno de los grandes cambios por tanto que precisan los programas de actividad física y deportiva extraescolar, es un cambio pedagógico, unido a la mejora en la formación de dichos profesionales y a una mejor interrelación con los responsables de la EF de los centros. En base a lo anterior, se detectan los siguientes hechos:

-Las actividades extraescolares fisico-deportivas se realizarán desconectadas de los programas de EF.

-Con respecto a estas actividades, parece ser que solo se centran en el ámbito deportivo, usando técnicos con formación deportiva en estos deportes.

-La formación de los monitores deportivos en algunos casos es sumamente deficitaria en pedagogía-didáctica, para desarrollar los programas de forma óptima.

-Predominan la oferta deportiva y competitiva frente a la expresiva y recreativa.

Desde la actual situación, sería necesario exigir a las administraciones unos programas de formación adecuado a las necesidades que se plantean y un presupuesto especial para el desarrollo de estas actividades de formación.

A continuación, voy a establecer la relación con el currículum. El currículum es definido según el artículo 6 de la Ley 2/2006 deEducación, como el conjunto de objetivos, competencias básicas, contenidos, métodos pedagógicos y criterios de evaluación de cada una de las enseñanzas reguladas en la LOE. Así pues, vamos a ver la relación con cada uno de los elementos del currículum.

\section{Relación con los Objetivos.}

La relación se establece con los siguientes objetivos, descritos en el Real Decreto 1631/2006, por el que se establecen las enseñanzas mínimas para la Educación Secundaria Obligatoria:
6. Realizar actividades físico-deportivas en el medio natural que tengan bajo impacto ambiental, contribuyendo a su conservación.

Supone aprovechar el entorno natural y sus posibilidades para la realización de actividades físicas, favoreciendo actitudes de respeto, cuidado y protección del medio. El docente deberá conocer el medio natural del municipio, tanto el más cercano como el más próximo, y aprovechará esto para trabajar el bloque de $\mathrm{AFMN}, .$.

8. Mostrar habilidades y actitudes sociales de respeto, trabajo en equipo y deportividad en la participación en actividades, juegos y deportes, independientemente de las diferencias culturales, sociales y de habilidad.

Podemos conseguir un hábito duradero en los alumnos de práctica de actividad física, y a partir de aquí, transferir estas actividades a otro tiempo didáctico, practicando fuera de la escuela. Las posibilidades que brinda el municipio serán imprescindibles para este objetivo.

-Relación con los Contenidos.

Dentro de los distintos bloques de contenidos, unos más que otros tendrán mayor implicación en el municipio la práctica de actividad física, así como los recursos naturales y materiales que este nos ofrece. A continuación reflejo aquellos que considero más relacionada.

Condición Física y Salud: Este contenido, muy relacionado con algunos de los objetivos y lo podemos implicar con el municipio y las posibilidades que éste les brinda para desarrollar su condición física individual en horas extraescolares.

Juegos y Deportes: Este contenido constituye una motivación que favorece el acercamiento natural a la práctica normalizada de la actividad física. Existe en el juego y en el deporte una respuesta a diferentes situaciones vivencias del alumno, y a través de las mismas se integra no sólo el espacio escolar, sino la calle, el barrio y, en general, el entorno, asumiendo diferentes manifestaciones populares y tradicionales.

Debe entenderse la práctica lúdica vinculada a la cultura circundante, que en el caso de la Comunidad Andaluza, aporta multitud de tradiciones y manifestaciones propias de indudable riqueza. Por tanto, hay que tener en cuenta la importancia de que los alumnos conozcan y practiquen Juegos Tradicionales, como vinculo y disfrute de patrimonio cultural de nuestra Comunidad.

Adquirir una elemental cultura deportiva, conociendo la utilización, de las instalaciones de su entorno próximo, así como respetar y conocer los valores educativos del deporte en todos sus ámbitos, deben ser contenidos de esta etapa educativa.

Haré un mayor hincapié en este contenido por considerar que está mucho más implicado en este aspecto que ahora nos ocupa (la práctica de actividad física en el municipio) ya que a partir de éste podemos conocer de una forma más directa el tipo de actividad que se ha realizado en nuestro municipio así como la que se realiza hoy día.

La importancia que el deporte ha adquirido en nuestra sociedad supera al ámbito de la práctica, para convertirse en un fenómeno social de masas que llega acondicionar el tipo y la modalidad deportiva que a los alumnos y alumnas les motiva practicar. La adecuación entre los contenidos a enseñar y el entorno socio-cultural en que se desarrollan los alumnos y alumnas, marcará las actividades de enseñanza- aprendizaje en este contenido.

En la elección de las habilidades especificas a enseñar, debe jugar un papel fundamental la observación de los juegos y deportes del entorno geográfico donde se ubica el centro y donde habita el alumno, por lo que es un buen procedimiento didáctico para el descubrimiento de los diversos factores culturales, económicos y políticos que giran en torno a ellos. Esta elección de actividades próximas al entorno hará que estos aprendizajes funcionales para los alumnos y, sobre todo, los vinculará a su comunidad.

Dentro de este contenido, desde nuestra perspectiva, podemos considerar los siguientes objetivos a conseguir con éste con1enldo en relación directa:

Procedimiento: 
-Practicar los diferentes juegos y deportes individuales, colectivos y alternativos, más habituales en el entorno, respetando sus normas y reglas.

-Práctica de juegos y/o deportes autóctonos.

-Práctica de juegos y deportes individuales y colectivos en el entorno natural con materiales no convencionales.

Conceptos:

-Conocimiento de los deportes más conocidos y/o practicados en su entorno, tanto individuales como colectivos.

-Localización de juegos y deportes autóctonos.

-Comparación entre las posibilidades del entono próximo para la práctica deportiva y las del entorno medio o lejano conocido.

-Conocimiento de los recursos disponibles para la práctica deportiva Instalaciones, material, entidades, asociaciones.

Actitudes:

-Valorar la práctica deportiva en su entorno inmediato

- Valorar los juegos y deportes autóctonos corno parte integrante de su cultura.

Las Actividades el Medio Natural: La Educación Física debe incorporar contenidos que sensibilicen a los alumnos sobre el medio natural, fomenten sus conocimientos y respeto y favorezcan su disfrute. Los alumnos deberán ser conscientes del entorno más próximo que les rodea y hacer cumplir en éste las normas de seguridad, protección y de precauciones a tener en cuenta en la utilización y disfrute del medio natural.

Será imprescindible conocer al municipio, sus recursos materiales, como posibilidades de lugares para la práctica de actividades como orientación, acampada, cabuyería, instalaciones, elementales de fortuna

Aprovechar los recursos propios de cada municipio para desarrollar este contenido en función de las posibilidades intrínsecas del municipio, enriqueciendo aún más este contenido. Y es que de las múltiples actividades que pueden realizarse en el marco natural, habrá que escoger aquellas que no sólo sean adecuadas a las capacidades e intereses de los alumnos como pudieran ser las acampadas, actividades de iniciación a la escalada, excursiones é itinerarios ecológicos o culturales, juegos y carreras de orientación, senderismo a pie, sino que también se adecuen a las posibilidades de nuestro entorno próximo o medio, en zonas concretas del Municipio o Comunidad, notablemente rica en espacios naturales.

-Relación con las Orientaciones Metodológicas.

A continuación presento toda una serie de orientaciones metodológicas que tienen como objetivo implicar las actividades, contenidos y objetivos curriculares con el municipio en el que sita el centro.

- Deberá proponerse una variada gama de actividades que puedan practicarse en el entorno, favoreciendo así trasferencias válidas a posteriores etapas educativas y diferentes momentos de ocio y recreación.

- La práctica de la educación física no se reduce solamente a momentos considerados escolares, sino que se extiende a diferentes momentos vitales del alumno. Es un proceso en el que intervienen elementos formales e informales de gran influencia social. El juego, el teatro, la danza, las adaptaciones deportivas, etc., son componentes curriculares que pertenecen a diferentes tiempos pedagógicos que es necesario asumir desde el planteamiento general del proyecto de Centro, de forma que exista una conjunción entre las diferentes actividades escolares con otras de tipo complementario y fuera del horario lectivo que también debe tener carácter formativo o incluirse coherentemente en el proyecto curricular.

- Las actividades propuestas deben adecuarse a las diferencias climáticas y la adecuación, duración e intensidad de las mismas, debe adaptarse a los hábitos sociales y costumbres alimentarias de nuestra Comunidad. La práctica de Actividad Física en un municipio perteneciente al norte de España no va a ser la misma que la que se practica en el Sur y una de las variables influyentes en esle hecho será la diferente afectación climática, en una y otra, así como los hábitos sociales y costumbres alimentarias, aspectos a conocer por él, programados para adecuar la actividad física a estos hechos.

- El desarrollo curricular de la actividad física debe tener en cuenta el medio en el que se realiza y el grupo de alumnos al que se dirige. Así se atenderá especialmente aspectos expresivos y creativos en medios culturalmente desfavorecidos. En medios urbanos, la práctica deportiva se adaptará en función del entorno, prestando especial atención al mantenimiento y mejora de la salud en ambientes contaminados. La intervención en propuestas comunes que diferentes soluciones con alumnos con problemas físicos y psíquicos, etc., son cuestiones que exigen una permanente adaptación curricular en función de sus características y peculiaridades.

- Referido a los contenidos de la habilidad Específica asociada a Juegos y Deportes y a la E.C., la programación de las actividades deportivas irá en función de las motivaciones del alumnado así como de las condiciones del entorno. Se tratará de una Iniciación Deportiva que progresivamente se va haciendo más específica para que su aplicación a Juegos y Deportes dé respuesta a una triple vertiente:

-Lúdico-deportiva

-Especializadora.

-Autóctona.: Se valorarán más los factores de inserción, en la Comunidad, el análisis y valor social de la propia práctica de un juego o deporte que tiene una significación en el patrimonio colectivo.

- La utilización del entorno próximo como contexto de aprendizaje. La utilización del entono próximo como contexto de aprendizaje, llevará a los alumnos a la comprensión del hecho de que las actividades físicas y deportivas pueden ser realizadas en diferentes entornos, que al mismo tiempo les permite la valoración del patrimonio natural y contribuir a su conservación y mejora posibilitando su uso y disfrute.

\section{Referencias Bibliográficas.}

Blández, J. (1995). La utilización del material y del espacio en educación física. Barcelona: Inde.

Blázquez, D. (1994). Los recursos en el currículum. Didáctica General. Alcoy: Marfil.

Contreras, O. (1998). Didáctica de la Educación Física: un enfoque constructivista. Barcelona: Inde.

De Lucas Heras, J.M. (2005). La dirección técnica de la Actividad Física y Deportiva como espacio profesional de los Licenciados en CC.A.F.D. Revista Española de Educación Física y deportes, 2, 2966.

Decreto 155/1997, de 10 de junio, por el que se regula la cooperación de las entidades locales con la Administración de la Junta de Andalucía en materia educativa. (BOJA: 15-07-97)

Decreto $27 / 1988$, de 10 de febrero, por el que se regulan las asociaciones de padres de alumnos de centros docentes no universitarios en el ámbito de la Comunidad Autónoma de Andalucía. (BOJA: 01- 03-88)

Decreto 200/1997, de 3 de septiembre, por el que se aprueba el Reglamento Orgánico de los IES.

Fernández Truán, J.C y Ruíz Fuster, M. (1997). Los materiales didácticos de educación física. Sevilla. Wanceulen.

Ley 13/1980 de 21 de Marzo (modificada en 5 de Octubre de 1990) de Cultura Física.

Ley Reguladora de Bases del Régimen Local de 2 de Abril de 1985.

Ley 10/1990 de 15 de Octubre, del Deporte.

Martínez del Castillo, J.; Fraile, A.; Puig, N. y Boixeda, A. (1991). Encuesta sobre estructura ocupacional del deporte en los sectores del entrenamiento, docencia, animación y dirección. Madrid: Consejo Superior de Deportes.

Ministerio de Educación y Ciencia (1992). Área de Educación Física. Primaria. Madrid: SGT.

Mena, B. (1997). Didáctica y Currículum Escolar. Salamanca: Anthema.

Orden de 11 de noviembre de 1997, por la que se regula el voluntariado y la participación de las Entidades Colaboradoras en Actividades Educativas Complementarias y Extraescolares de los Centros Docentes. (BOJA: 27-11-97)

Real Decreto 1631/2006, por el que se establecen las enseñanzas mínimas para la Educación Secundaria Obligatoria.

Rebollo Rico, S. (1996). El espacio deportivo no convencional: aprovechamiento de los recursos naturales y urbanos. Inédito: Granada.

Rodríguez, J. (2003). El municipio y la práctica de Actividad Física. Inédito: Granada.

Ruiz Munuera, F.C. (2002). El municipio y la práctica de Actividad Física. Madrid: Mad. 